\title{
Protective effect of Porphyra yezoensis glycoprotein on D-galactosamine-induced cytotoxicity in Hepa 1c1c7 cells
}

\author{
JEONG-WOOK CHOI, YOUNG-MIN KIM, SU-JIN PARK, IN-HYE KIM and TAEK-JEONG NAM \\ Department of Food and Life Science, Pukyong National University, Nam-gu, Busan 608-737, Republic of Korea
}

Received April 7, 2014; Accepted December 19, 2014

DOI: $10.3892 / \mathrm{mmr} .2015 .3244$

\begin{abstract}
The present study aimed to examine the signaling pathways and enzyme activity associated with the protective effect of Porphyra yezoensis glycoprotein (PYGP) on D-galactosamine (D-GaIN)-induced cytotoxicity in Hepa 1c1c7 cells. D-GaIN is commonly used to induce hepatic injury models in vivo as well as in vitro. PYGP was extracted from Porphyra yezoensis, a red algae distributed along the coasts of Republic of Korea, China and Japan. In the present study, Hepa 1clc7 cells were pre-treated with PYGP (20 and $40 \mu \mathrm{g} / \mathrm{ml}$ ) for $24 \mathrm{~h}$ and then the media was replaced with D-GaIN (20 mM) and PYGP (20 and $40 \mu \mathrm{g} / \mathrm{ml}$ ). The results demonstrated that D-GaIN induced Hepa 1c1c7 cell death and pretreatment with PYGP was found to attenuate D-GaIN toxicity. In addition, D-GaIN decreased the antioxidant activity and increased lipid peroxidation processes; however, pre-treatment with PYGP reduced the generation of lipid peroxidation products, such as thiobarbituric acid reactive substances, as well as increased the activity of antioxidant enzymes, including superoxide dismutase, catalase and glutathione-s-transferase (GST). PYGP was shown to suppress the overexpression of extracellular signal-regulated kinase, c-jun N-terminal kinase and p38 mitogen-activated protein kinase (MAPK) phosphorylation induced by D-GaIN. Furthermore, PYGP increased the protein expression of nuclear factor erythroid 2-related factor 2 (Nrf2), quinine oxidoreductase 1, GST and heme oxygenase 1 protein expression. These results suggested that PYGP had cytoprotective effects against D-GaIN-induced cell damage, which may be associated with MAPKs and the Nrf2 signaling pathway.
\end{abstract}

\section{Introduction}

Liver disease is a prevalent medical condition which has numerous etiologies, including chemical exposure, alcohol, lipid

Correspondence to: Professor Taek-Jeong Nam, Department of Food and Life Science, Pukyong National University, 599-1 Daeyeon 3-dong, Nam-gu, Busan 608-737, Republic of Korea

E-mail: namtj@pknu.ac.kr

Key words: nuclear factor erythroid 2-related factor 2, mitogen-activated protein kinase, galactosamine, Porphyra yezoensis peroxidative products and viral infection (1). To date, several types of medications have been investigated for the treatment of liver diseases (2).

D-galactosamine (D-GaIN) is a well-known inducer of hepatic injury in vitro and in vivo. D-GaIN treatment results in the loss of uridine 5'-triphosphate, uridine 5'-diphosphate and uridine 5'-monophosphate as well as inhibition of RNA and protein synthesis (3). In addition, D-GaIN-induced oxidative stress is generated through reactive hydroxyl radical damage to the cell membrane via stimulation of lipid peroxidation (4). Several studies have shown that D-GaIN-induced hepatocyte death was mediated through mitogen-activated protein kinase (MAPK) and nuclear factor erythroid 2-related factor 2 (Nrf2) signaling (5-7).

Nrf2 is a transcription factor which targets certain genes, including those for nicotinamide adenine dinucleotide phosphate hydrogen (NADPH), quinine oxidoreductase 1 (Nqo1), glutathione (GSH) synthesis and glutathione-s-transferase (GST) (8); in addition, Nrf2 has been shown to have protective effects against oxidative stress (9). During oxidative stress, Nrf2 translocates to the nucleus from the cytosol, and as a result, antioxidant enzymes become upregulated and oxidative stress damage decreases (10).

MAPKs include c-jun N-terminal kinase (JNK), p38 MAPK and extracellular signal-regulated kinase (ERK). These proteins have been reported to be phosphorylated via GaIN-induced oxidative stress (5). Of note, activated-JNK induces hepatocyte death and apoptosis via activation of caspase-3 as well as liver cell necrosis (11).

Superoxide dismutase (SOD) and catalase (CAT) are important cellular defense systems which transform superoxide into oxygen and hydrogen peroxide for detoxification (12). In addition, GSTs are a superfamily of enzymes that protect against chemical toxicity and oxidative stress $(13,14)$. Porphyra (P.) yezoensis is a red algae found along the coasts of Korea, China and Japan. In the present study, the protective effect of Porphyra yezoensis glycoprotein (PYGP) on D-GaIN-induced cytotoxicity was investigated in Hepa 1clc7 cells.

\section{Materials and methods}

Preparation of PYGP. P. yezoensis was purchased in 2013 in the Republic of Korea (Shuyup, Busan, Korea). P. yezoensis powder ( $40 \mathrm{~g}$ ) was suspended in 11 distilled water and stirred for $4 \mathrm{~h}$ at room temperature. The suspension was centrifuged 
at $3,000 \mathrm{x}$ g at $4^{\circ} \mathrm{C}$ for $20 \mathrm{~min}$ and vacuum filtered, followed by the addition of triple volumes (total quantity of filtrate $\mathrm{x} 3$ ) of ethanol. Following $24 \mathrm{~h}$, the solution was filtered and concentrated using rotary evaporation at $40^{\circ} \mathrm{C}$. The concentrated solution was divided into $1.5-\mathrm{ml}$ tubes, freeze-dried and stored at $-70^{\circ} \mathrm{C}$ until further use.

Cell culture. Normal mouse Hepa 1c1c7 hepatocyte cells (no. 22026) were purchased from the Korean Cell Line Bank (Seoul, Korea). Cells were maintained at $37^{\circ} \mathrm{C}$ in a $5 \% \mathrm{CO}_{2}$ humidified atmosphere. Hepa 1c1c-7 cells were cultured in minimum essential medium $\alpha$ (Gibco-BRL, Grand Island, NY, USA) without nucleosides supplemented with $10 \%$ fetal bovine serum (FBS; Hyclone Laboratories, Inc., Logan, UT, USA) and antibiotics (Gibco-BRL). The medium was replaced every two days.

Cell proliferation. Hepa 1c1c7 cell proliferation was measured using a CellTiter 96 aqueous non-radioactivity cell proliferation assay (Promega Corp., Madison, WI, USA). This assay determines cell proliferation based on the cleavage of 3-(4,5-dimethylthiazol-2-yl)-5-(3-carboxymethoxy-phenyl)-2-(4-sulfonyl)-2H-tetrazolium (MTS) into a formazan product, which is soluble in tissue culture medium. Hepa 1c1c7 cells were seeded onto 96-well plates at a density of $1.5 \times 10^{3}$ cells/well in $100 \mu 1$ medium. Cells were cultured for $24 \mathrm{~h}$, following which the medium was replaced with serum-free medium (SFM) containing PYGP (20 or $40 \mu \mathrm{g} / \mathrm{ml})$ for $24 \mathrm{~h}$. PYGP-treated Hepa 1c1c7 cells were then exposed to $20 \mathrm{mM}$ D-GaIN (Sigma-Aldrich, St. Louis, MO, USA) for $24 \mathrm{~h}$. Subsequently, cells were incubated in MTS solution for $30 \mathrm{~min}$ at $37^{\circ} \mathrm{C}$. Cell proliferation was measured at $490 \mathrm{~nm}$ using a Benchmark Plus 10730 microplate reader (Benchmark; Bio-Rad Laboratories, Inc., Hercules, CA, USA).

Estimation of dehydrogenase release. Hepa 1c1c7 cell injury was quantitatively assessed via determination of lactate dehydrogenase (LDH), which is released from damaged or destroyed cells. Hepa 1c1c7 cells were seeded onto 96-well plates at a density of $1.5 \times 10^{3}$ cells/well in $100 \mu \mathrm{l}$ medium. Cells were cultured for $24 \mathrm{~h}$, following which the SFM was replaced with PYGP (20 or $40 \mu \mathrm{g} / \mathrm{ml}$ ) for $24 \mathrm{~h}$. Hepa 1clc7 cells were then exposed to either D-GaIN (20 mM) with PYGP (20 or $40 \mu \mathrm{g} / \mathrm{ml}$ ) for $24 \mathrm{~h}$. LDH release was measured using an LDH cytotoxicity assay kit according to the manufacturer's instructions (Cayman Chemical Co., Ann Arbor, MI, USA). Absorbance was then measured at $490 \mathrm{~nm}$ using a Benchmark Plus 10730 microplate reader (Bio-Rad Laboratories, Inc.).

Lipid peroxidation measurement. Cells were collected in lysis buffer (phosphate-buffered saline, $0.05 \%$ butyl hydroxyl toluene; Cell Biolabs Inc., San Diego, CA, USA) and homogenized on ice using a thiobarbituric acid reactive substances (TBARS) assay kit (Cell Biolabs, Inc.) according to the manufacturer's instructions. Absorbance was then measured at $532 \mathrm{~nm}$ using a Benchmark Plus 10730 microplate reader (Bio-Rad Laboratories, Inc.).

Western blot analysis. Hepa 1c1c7 cells were plated onto $100-\mathrm{mm}$ dishes. Cells were cultured until they reached
$60-80 \%$ confluence and were then pre-treated with PYGP (20 or $40 \mu \mathrm{g} / \mathrm{ml}$ ) for $24 \mathrm{~h}$. Cells were then exposed to D-GaIN (20 mM) with PYGP (20 or $40 \mu \mathrm{g} / \mathrm{ml}$ ) for $24 \mathrm{~h}$. Cells were washed with ice-cold phosphate-buffered saline (PBS; 0.15 M sodium phosphate, 0.15 M sodium chloride, pH 7.4; Gibco-BRL), following which lysis buffer [20 mM Tris-base (pH 7.5), $150 \mathrm{mM} \mathrm{NaCl}$, $0.25 \%$ Na-deoxycholate, $1 \mathrm{mM}$ EDTA, $1 \mathrm{mM}$ ethylene glycol tetraacetic acid, $1 \%$ Triton X-100 from iNtRON Biotechnology, Seoul, Korea; containing 2.5 M sodium pyrophosphate, $1 \mathrm{mM}$ $\beta$-glycerophosphate, $1 \mathrm{mM} \mathrm{Na} \mathrm{VO}_{4}, 1 \mu \mathrm{g} / \mathrm{ml}$ aprotinin, $1 \mu \mathrm{g} / \mathrm{ml}$ leupeptin, $1 \mu \mathrm{g} / \mathrm{ml}$ pepstatin $\mathrm{A}$ and $1 \mathrm{mM}$ phenylmethylsulfonyl fluoride from Sigma-Aldrich] was added. Protein content was determined using a bicinchoninic acid protein assay kit (Pierce Biotechnology, Inc., Rockford,IL, USA). Proteins were separated using 10-15\% SDS-PAGE and then transferred to a polyvinylidene fluoride membrane (Millipore, Billerica, MA, USA). The transferred membrane was blocked at room temperature with $1 \%$ bovine serum albumin (BSA) in Tris-buffered saline with Tween 20 [TBS-T; 10 mM Tris- $\mathrm{HCl}$ (pH 7.5), $150 \mathrm{mM} \mathrm{NaCl}$ and $0.1 \%$ Tween 20] and then incubated, with agitation, with the indicated primary antibodies: Rabbit anti-mouse ERK immunoglobulin $\mathrm{G}(\mathrm{IgG})$ polyclonal antibody [diluted 1:1,000 with BSA/TBS-T; incubated for $4 \mathrm{~h}$ at room temperature (RT)], rabbit anti-phosphorylated (p)-ERK IgG polyclonal antibody (diluted 1:1,000 with BSA/TBS-T; incubated for $4 \mathrm{~h}$ at RT), mouse anti-mouse JNK IgG monoclonal antibody (diluted 1:1,000 with BSA/TBS-T; incubated for $4 \mathrm{~h}$ at RT), mouse anti-mouse p-JNK IgG monoclonal antibody (diluted 1:1,000 with BSA/TBS-T; incubated for $4 \mathrm{~h}$ at RT), rabbit anti-mouse p38 IgG polyclonal antibody (diluted 1:1,000 with BSA/TBS-T; incubated for $4 \mathrm{~h}$ at RT), mouse anti-mouse p-p38 IgG monoclonal antibody (diluted 1:1,000 with BSA/TBS-T; incubated for $4 \mathrm{~h}$ at RT), rabbit anti-mouse Nrf2 IgG polyclonal antibody (diluted 1:1,000 with BSA/TBS-T; incubated for $4 \mathrm{~h}$ at RT), goat anti-mouse Nqo1 IgG polyclonal antibody (diluted 1:1,000 with BSA/TBS-T; incubated for $4 \mathrm{~h}$ at RT), mouse anti-mouse GST IgG monoclonal antibody (diluted 1:1,000 with BSA/TBS-T; incubated for $4 \mathrm{~h}$ at RT), and rabbit anti-mouse heme oxygenase (HO)-1 IgG polyclonal antibody (diluted 1:1,000 with BSA/ TBS-T; incubated for $4 \mathrm{~h}$ at RT), which were all purchased from Santa Cruz Biotechnology, Inc. (Dallas, TX, USA). The secondary antibody was a peroxidase-conjugated goat, mouse and rabbit antibody (1:10,000; GE Healthcare, Little Chalfont, UK). Super Signal West Pico Stable Peroxide Solution and the Super Signal West Pico Luminol/Enhancer solution (Thermo Fisher Scientific, Rockford, IL, USA) were then added and the signal was monitored using X-ray film (Kodak, Rochester, NY, USA) and a developer and fixer twin pack (Kodak).

Antioxidant enzyme measurement. SOD (Superoxide dismutase assay kit; Cayman Chemical Co., Ann Arbor, MI, USA), CAT (Catalase assay kit; Cayman Chemical Co.) and GST (Glutathione s-transferase assay kit; Cayman Chemical Co.) activities of Hepa 1clc7 cells were measured according to the manufacturer's instructions. Absorbance was then measured using a Benchmark microplate reader (Benchmark Plus 10730; Bio-Rad Laboratories, Inc.).

Statistical analysis. Values are presented as the mean \pm standard deviation and data were analyzed with SPSS 10.0 

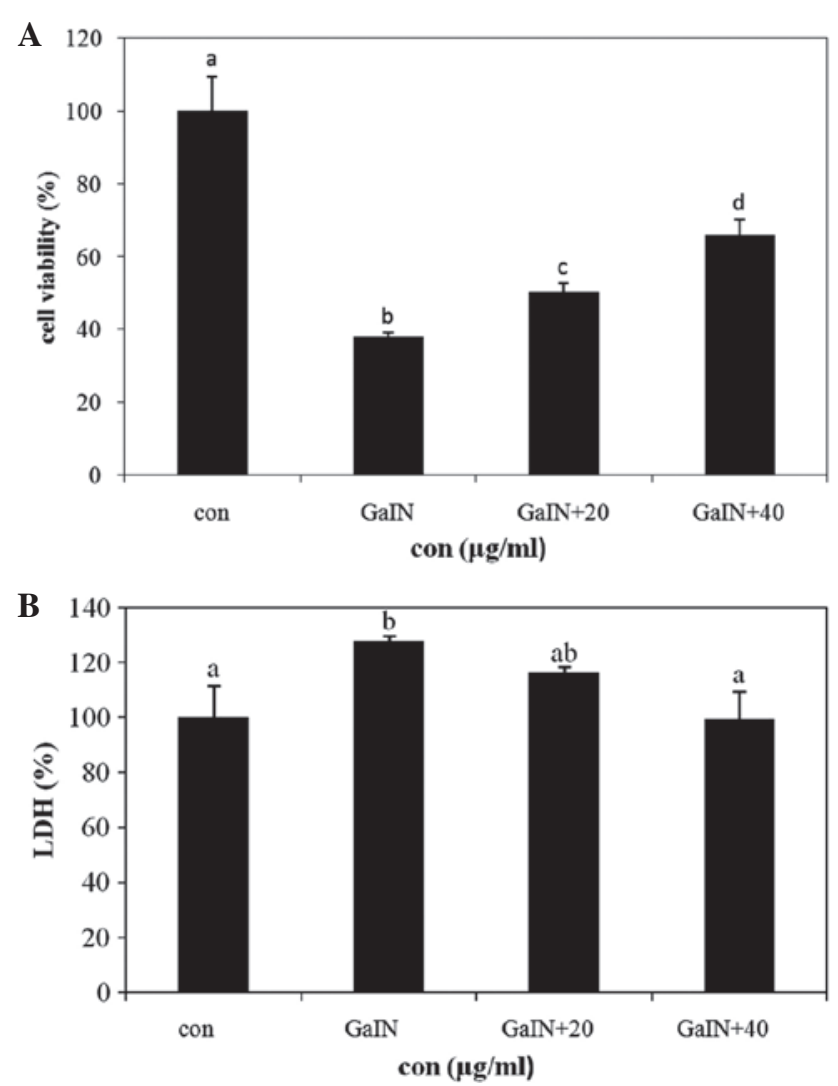

Figure 1. Effect of PYGP on D-GaIN-induced cytotoxicity in Hepa 1c1c7 cells. Cells were pre-treated with PYGP ( 20 and $40 \mu \mathrm{g} / \mathrm{ml}$ ) for $24 \mathrm{~h}$ and then treated with $20 \mathrm{mM}$ D-GaIN with PYGP (20 and $40 \mu \mathrm{g} / \mathrm{ml}$ ) for $24 \mathrm{~h}$. (A) Cell viability and (B) released LDH levels were determined following D-GaIN-induced cell cytotoxity. Values are presented as the mean \pm standard deviation. Values with different letters are significantly different $(\mathrm{P}<0.05)$. PYGP, Porphyra yezoensis glycoprotein; D-GaIN, D-galactosamine; LDH, lactate dehydrogenase; con, control.

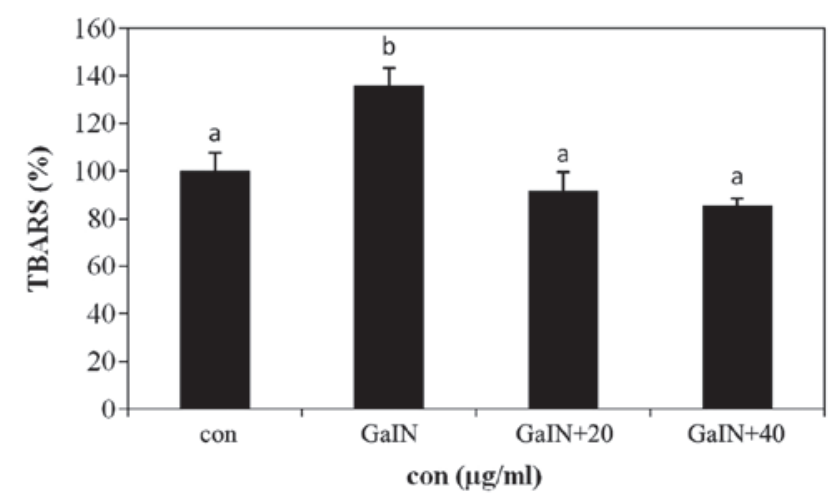

Figure 2. Effect of PYGP on D-GaIN-induced lipoperoxidation. Hepa 1c1c7 cells were pre-treated with PYGP $(20$ and $40 \mu \mathrm{g} / \mathrm{ml})$ for $24 \mathrm{~h}$ and then administered $20 \mathrm{mM}$ D-GaIN with PYGP $(20$ and $40 \mu \mathrm{g} / \mathrm{ml})$ for $24 \mathrm{~h}$. Cell pellets were then collected using $1 \mathrm{X}$ butyl hydroxyl toluene homogenized on ice using a TBARS assay kit. Values are presented as the mean \pm standard deviation. Values with different letters are significantly different $(\mathrm{P}<0.05)$. PYGP, Porphyra yezoensis glycoprotein; D-GaIN, D-galactosamine; TBARS, thiobarbituric acid reactive substances; con, control.

software (SPSS, Inc., Chicago, IL, USA) using an analysis of variance followed by a Duncan's multiple range test. $\mathrm{P}<0.05$ was considered to indicate a statistically significant difference between values.

\section{Results}

Protective effects of PYGP against D-GaIN-induced injury in Hepa 1c1c7 cells. The protective effect of PYGP against D-GaIN-induced hepatotoxicity in normal mouse liver cells was measured using the MTS cell viability assay. Cells were pre-treated with PYGP for $24 \mathrm{~h}$ and then incubated with D-GaIN and PYGP for $24 \mathrm{~h}$. The results of the MTS assay showed that D-GaIN treatment induced Hepa 1c1c7 cell death, whereas pretreatment with PYGP significantly attenuated the cytotoxic effects of D-GaIN (P<0.05; Fig. 1A).

LDH is a soluble enzyme located in the cytosol, which is released into the surrounding culture medium upon cell damage $(15,16)$. The results of the present study demonstrated that the D-GaIN-only treatment group showed increased LDH levels compared with those of the control group; by contrast, the PYGP pre-treatment groups showed significantly decreased LDH enzyme release compared with that of the D-GaIN-only treatment group $(\mathrm{P}<0.05$; Fig. 1B).

Inhibitory effect of Hepa 1c1c7 cells on TBARS production using PYGP. Lipid peroxidation induces TBARS generation and cell damage through oxidative stress; in addition, TBARS and lipid peroxide produced during oxidative stress may cause or aggravate diseases associated with aging and hepatotoxicity (17). The results of the present study revealed that levels of TBARS were increased in the D-GaIN-only treatment group compared with those of the control group. In addition, pre-treatment with PYGP significantly inhibited the D-GaIN-induced increase in TBARS levels ( $\mathrm{P}<0.05$; Fig. 2).

Increased antioxidant enzyme activity in Hepa 1clc7 cells in the presence of PYGP. CAT, SOD and GST are the primary defensive enzymatic antioxidants in eukaryotic cells (18). The activity levels of these enzymes were all found to be significantly inhibited in the D-GaIN-only treatment group compared with those in the control group. By contrast, the PYGP pre-treatment group showed increased antioxidant enzyme activity compared with that in the D-GaIN-only treatment group $(\mathrm{P}<0.05$; Fig. 3$)$.

Effect of PYGP pre-treatment on GaIN-induced expression and phosphorlyation of via MAPKs. ERK, JNK and p38 MAPK are known to be phosphorylated and activated in response to D-GaIN (11). In the present study, the phosphorylation of each MAPK was examined using western blot analysis. The results revealed that PYGP suppressed the D-GaIN-induced activation of each MAPK; in addition, treatment with $40 \mu \mathrm{g} / \mathrm{ml}$ PYGP was observed to have a more suppressive effect compared with that of $20 \mu \mathrm{g} / \mathrm{ml}$ PYGP. However, total ERK, JNK and p38 MAPK protein expression levels were not altered following treatment with D-GaIN or PYGP pre-treatment (Fig. 4).

PYGP upregulates the Nrf2 signaling pathway. Nrf2 has previously been reported to increase the expression of various oxidant defense proteins, including HO-1, Nqo1 and GST $(7,8)$. In the present study, western blot analysis was used to examine the expression of these proteins following D-GaIN-induced cell injury and pre-treatment with PYGP. Cells exposed to D-GaIN 

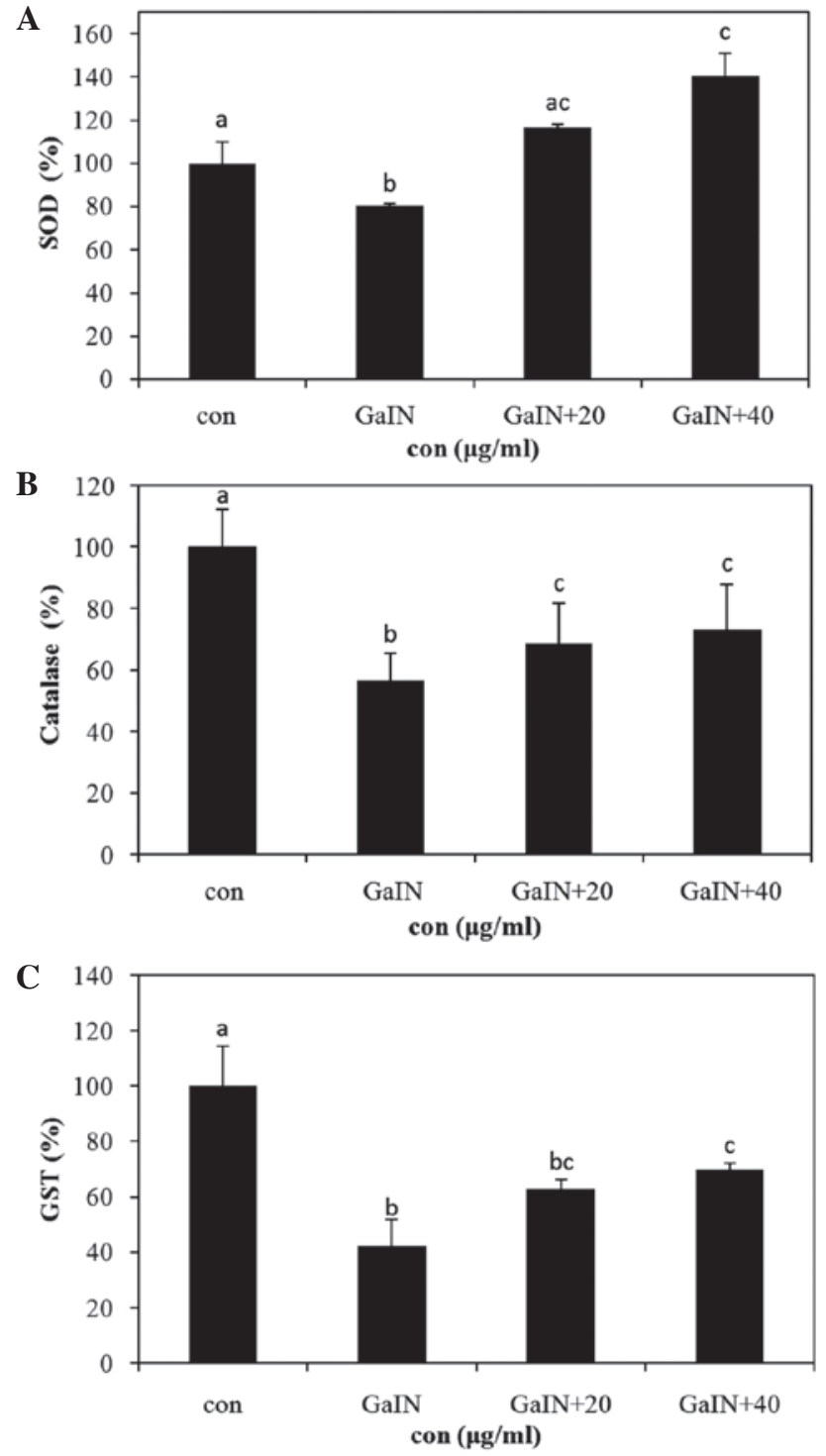

Figure 3. Effect of PYGP on the activity of the antioxidant enzymes SOD, CAT and GST following D-GaIN-induced cytotoxicity. Hepa 1c1c7 cells were pre-treated with PYGP (20 and $40 \mu \mathrm{g} / \mathrm{ml})$ for $24 \mathrm{~h}$ and then administered $20 \mathrm{mM}$ D-GaIN with PYGP (20 and $40 \mu \mathrm{g} / \mathrm{ml}$ ) for $24 \mathrm{~h}$. (A) SOD, (B) CAT and (C) GST activity were determined. Values are presented as the mean \pm standard deviation. Values with different letters are significantly different $(\mathrm{P}<0.05)$. PYGP, Porphyra yezoensis glycoprotein; D-GaIN, D-galactosamine; con, control; SOD, superoxide dismutase; CAT, catalase; GST, glutathione s-transferase.

only showed reduced Nrf2, HO-1, Nqo1 and GST protein expression levels compared with those of the untreated cells. By contrast, the expression of these Nrf2-stimulated proteins following PYGP pre-treatment were significantly increased compared with those in the D-GaIN-only group (Fig. 5).

\section{Discussion}

In the present study, cytotoxic injury was induced in Hepa 1c1c7 cells using D-GaIN, which is a commonly used model for screening anti-hepatotoxic and anti-hepatoxic activities of drugs. D-GaIN-induced liver injury has previously been shown to closely resemble acute viral hepatitis $(19,20)$. D-GaIN has direct and indirect roles, which affect the oxidative stress

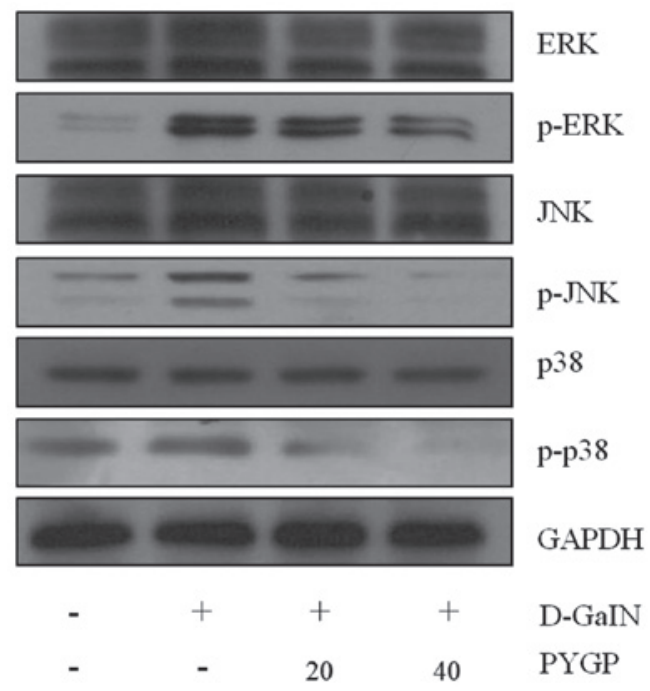

Figure 4. Effect of PYGP on D-GaIN-induced expression and phosphorylation of MAPKs in Hepa 1c1c7 cells. Cells were pre-treated with PYGP (20 and $40 \mu \mathrm{g} / \mathrm{ml}$ ) for $24 \mathrm{~h}$ and then administered $20 \mathrm{mM} \mathrm{D-GaIN}$ with PYGP (20 and $40 \mu \mathrm{g} / \mathrm{ml}$ ) for $24 \mathrm{~h}$. Cell pellets were then collected using lysis buffer and western blot analysis was performed in order to determine the total protein expression and phosphorylation levels of ERK, JNK and p38 MAPK. PYGP, Porphyra yezoensis glycoprotein; D-GaIN, D-galactosamine; MAPK, mitogen-activated protein kinase; ERK, extracellular signal-regulated kinase; JNK, c-jun N-terminal kinase; p-, phosphorylated.

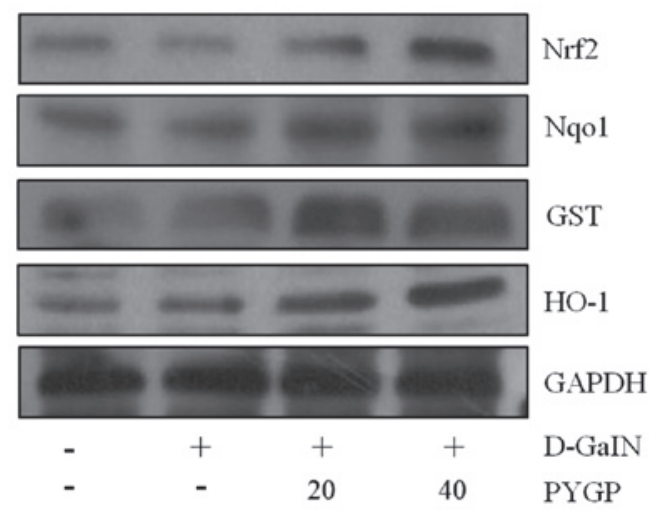

Figure 5. Effect of PYGP on D-GaIN-induced expression of Nrf2, Nqo1, GST and HO-1 in Hepa 1c1c7 cells. Cells were pre-treated with PYGP (20 and $40 \mu \mathrm{g} / \mathrm{ml}$ ) for $24 \mathrm{~h}$ and then administered $20 \mathrm{mM}$ D-GaIN with PYGP (20 and $40 \mu \mathrm{g} / \mathrm{ml}$ ) for $24 \mathrm{~h}$. Cell pellets were then collected using lysis buffer and western blot analysis was performed in order to determine protein expression levels of Nrf2, Nqol, GST and HO-1. PYGP, Porphyra yezoensis glycoprotein; D-GaIN, D-galactosamine; Nrf2, nuclear factor erythroid 2-related factor 2; Nqol, quinine oxidoreductase 1; GST, glutathione s-transferase; HO-1, heme oxygenase 1 .

properties of organs. Several studies have shown that D-GaIN induced changes in liver antioxidant enzyme levels (17,21-23). In addition, D-GaIN was reported to induce hepatotoxicity by inhibiting RNA and protein synthesis as well as reducing uridine 5'-triphosphate, uridine 5'-diphosphate and uridine 5'-monophosphate levels $(17,24,25)$. Increased LDH release into the medium as a result of cell damage is widely used as a measure of cytotoxicity (26). In the present study, D-GaIN treatment was found to induce cytotoxicity in Hepa 1c1c7 cells, the effect of which was attenuated in cells pre-treated with PYGP. 
Peroxidation of endogenous lipids is a major factor affecting the cytotoxic activity of D-GaIN (7). D-GaIN-induced oxidative stress damage is generally attributed to the formation of highly reactive hydroxyl radicals, such as superoxide anions, which stimulate lipid peroxidation and damage cell membranes $(4,27)$. In the present study, TBARS was significantly increased following D-GaIN-only treatment; however, pre-treatment with PYGP decreased the oxidative damage in cells, based on the decreased levels of TBARS compared to those in the D-GaIN-only group. SOD and CAT are first-line cellular antioxidant defense enzymes. SOD reacts with $\mathrm{O}_{2}$ in order to generate $\mathrm{H}_{2} \mathrm{O}_{2}$ and $\mathrm{H}_{2} \mathrm{O}$ (13), while CAT accelerates the dismutation reaction of $\mathrm{H}_{2} \mathrm{O}_{2}$ and the formation of $\mathrm{H}_{2} \mathrm{O}$ and $\mathrm{O}_{2}(28,29)$. GST binds to numerous different lipophilic drugs and chemicals; thus, it likely binds to D-GaIN and functions as an enzyme for GSH conjugation reactions (30). In the present study, lipid peroxidation induced by D-GaIN was measured; following $24 \mathrm{~h}$ of exposure to D-GaIN, there was a significant increase in TBARS levels compared with those of the control group. However, the PYGP pre-treatment groups showed reduced TBARS levels compared with those in the D-GaIN-only group. Furthermore, D-GaIN significantly decreased the activity levels of the antioxidant enzymes CAT, GST and SOD compared with those in the control group, while pretreatment with PYGP increased these enzyme levels compared with those in the D-GaIN-only treatment group.

The MAPK signaling pathway is an important signaling pathway which regulates tumor necrosis factor (TNF)- $\alpha$ expression; however, the detailed mechanism of this remains to be fully elucidated $(31,32)$. MAPKs have been confirmed to participate in regulating cytokine production in response to a broad range of stimuli $(33,34)$. MAPKs include three major proteins: ERK, JNK and p38 MAPK. These proteins have important biological roles in cell proliferation, differentiation, metabolism, survival and apoptosis (35). In the present study, levels of their activated forms, p-ERK, p-JNK, and p-p38 MAPK, were observed to be increased in the D-GaIN only treatment group compared with those in the untreated control group; however, PYGP pre-treatment attenuated the D-GaIN-mediated activation of ERK, JNK and p38 MAPK.

As a transcription factor, Nrf2 promotes the translation of genes which have a protective effect against oxidative/electrophilic stress $(36,37)$. Nrf2 exists as a binding repressor of kelch-like erythroid cell-derived protein with CNC homology-associated protein 1 (Keap 1) in the cytoplasm (38). In response to oxidative stress, Nrf2 dissociates from Keap 1 and translocates to the nucleus in order to induce an array of cytoprotective genes, including Nqo1, GST and HO-1 $(39,40)$. In the present study, western blot analysis revealed that Nrf2, Nqo1, GST and HO-1 expression levels were decreased in the presence of D-GaIN. However, PYGP pre-treatment increased Nrf2, Nqo1, GST and HO-1 expression levels compared with those in the D-GaIN-only treatment group. These results indicated that PYGP pre-treatment upregulated Nrf2 protein levels and stimulated the activity of antioxidants and phase II detoxifying enzymes.

In conclusion, the results of the present study demonstrated that PYGP reduced D-GaIN-induced hepatotoxicity in normal mouse Hepa 1c1c7 hepatocytes via upregulation of antioxidative enzymes, MAPKs and the Nrf2 pathway. These findings therefore indicated that PYGP may have potential for use for the prevention of hepatotoxicity.

\section{Acknowledgements}

The present study was supported by the Fishery Commercialization Technology Development Program through Korea Institute of Planning and Evaluation for Technology in Food, Agriculture, Forestry and Fisheries (iPET) funded by the Ministry of Oceans and Fisheries (MOF; 2012300734).

\section{References}

1. Hwang JM, Tseng TH, Tsai YY, Lee HJ, Chou FP, Wang CJ and Chu CY: Protective effects of baicalein on tert-butyl hydroperoxide-induced hepatic toxicity in rat hepatocytes. J Biomed Sci 12: 389-397, 2005.

2. Jose M, Javier C, Javier FP, et al: S-Adenosylmethionine in alcoholic liver cirrhosis: a randomized, placebo-controlled, double-blind, multicenter clinical trial. J Hepatol 30: 1081-1089, 1999.

3. MacDonald JR, Thayer KJ and White C: Inhibition of galactosamine cytotoxicity in an in vivo/in vitro hepatocellular toxicity model. Toxicol Appl Pharmacol 89: 269-277, 1987.

4. Sakaguchi $\mathrm{S}$ and Yokota K: Role of $\mathrm{Ca}^{2+}$ on endotoxin-sensitivity by galactosamine challenge: lipid peroxide formation and hepatotoxicity in zymosan primed mice. Pharmacol Toxicol 77: 81-86, 1995.

5. Hou CC, Huang CC and Shyur LF: Echinacea alkamides prevent lipopolysaccharide/D-galactosamine-induced acute hepatic injury through JNK pathway-mediated HO-1 expression. J Agric Food Chem 59: 11966-11974, 2011.

6. Cho HI, Park JH, Choi HS, Kwak JH, Lee DU, Lee SK and Lee SM: Protective mechanisms of acacetin against D-galactosamine and lipopolysaccharide-induced fulminant hepatic failure in mice. J Nat Prod 77: 2497-2503, 2014.

7. Das J, Ghosh J, Roy A and Sil PC: Mangiferin exerts hepatoprotective activity against D-galactosamine induced acute toxicity and oxidative/nitrosative stress via $\mathrm{Nrf2}-\mathrm{NF} \kappa \mathrm{B}$ pathways. Toxicol Appl Pharmacol 260: 35-47, 2012.

8. Rajesh KT, Kim HM, Sorachai S, Thomas WK, Masayuki Y and Shyam B: Identification of Nrf2-regulated genes induced by the chemopreventive agent sulforaphane by oligonucleotide microarray. Cancer Res 62: 5196-5203, 2002.

9. Martin E, Victoria B, Walter M, Andrea GL and Patricia GV: The Nrf2-Keap1 cellular defense pathway and heat shock protein 70 (Hsp70) response. Role in protection against oxidative stress in early neonatal unilateral ureteral obstruction (UUO). Cell Stress Chaperones 16: 57-68, 2011.

10. Dhakshinamoorthy S and Jaiswal AK: Functional characterization and role of INrf 2 in antioxidant response element-mediated expression and antioxidant induction of NAD (P) H:quinine oxidoreductase 1 gene. Oncogene 20: 3906-3917, 2001.

11. Nishioka H, Kishioka T, Iida C, Fujii K, Ichi I and Kojo S: Activation of mitogen activated protein kinase (MAPK) during D-galactosamine intoxication in the rat liver. Bioorg Med Chem Lett 16: 3019-3022, 2006.

12. Sally KN, Swapan KB, Gary KG, Paul M and Joe MM: The induction of human superoxide dismutase and catalase in vivo: A fundamentally new approach to antioxidant therapy. Free Radic Biol Med 40: 341-347, 2006.

13. Nirupama $M$ and Friedrich HM: reactive oxygen species: response of algal cells. J Plant Physiol 157: 183-193, 2000.

14. Chao ES, Dunbar D and Kaminsky LS: Intracellular lactate dehydrogenase concentration as an index of cytotoxicity in rat hepatocyte pri mary culture. Cell Biol Toxicol 4: 1-11, 1988.

15. Jauregui HO, Hayner NT, Driscoll JL, Williams-Holland R, Lipsky MH and Galletti PM: Trypan blue dye uptake and lactate dehydrogenase in adult rat hepatocytes-freshly isolated cells, cell suspension and primary mono layer cultures. In Vitro 17: 1100-1110, 1981.

16. Allocati N, Federici L, Masulli M and Di Ilio C: Glutathione transferases in bacteria. Febs J 276: L58-L75, 2009. 
17. Pushpavalli G, Kalaiarasi P, Veeramani C and Pugalendi KV: Effect of chrysin on hepatoprotective and antioxidant status in D-galactosamine-induced hepatitis in rat. Eur J Pharmacol 631: 36-41, 2010.

18. Halliwell B: Biochemistry of oxidative stress. Biochem Soc Trans 35: 1147-1150, 2007.

19. Decker K and Keppler D: Galactosamine induced liver injury. Proq Liver Dis 4: 183-199, 1972.

20. Nakagiri R, Hashizume E, Kavahashi S, Sakai Y and Kamiya T: Suppression by hydrangea Dulcis folium of $\mathrm{D}$-galactosamine-induced liver injury in vitro and in vivo. Biosci Biotechnol Biochem 67: 2641-2643, 2003.

21. Han KH, Hashimoto N, Shimada K, Sekikawa M, Noda T, Yamauchi H, Hashimoto M, Chiji H, Topping DL and Fukushima M: Hepatoprotective effects of purple potato extract against D-galactosamine-induced liver injury in rats. Biosci Biotechnol Biochem 70: 1432-1437, 2006.

22. Shi Y, Sun J,He H, Guo HandZhang S: Hepatoprotective effects of Ganoderma lucidum peptides against D-galactosamine-induced liver injury in mice. J Ethnopharmacol 117: 415-419, 2008.

23. Lim HK, Kim HS, Choi HS, Oh S, Jang CG, Choi J, Kim SH and Chang MJ: Effects of acetylbergenin against D-galactosamine-induced hepatotoxicity in rats. Pharmacol Res 42: 471-474, 2000.

24. Tang XH, Gao L, Gao J, Fan YM, Xu LZ, Zhao XN and Xu Q: Mechanisms of hepatoprotection of Terminalia catappa $\mathrm{L}$. extract on D-galactosamine-induced liver damage. AM J Chin Med 32: 509-519, 2004

25. Aristatile B, Al-Numair KS, Al-Assaf $\mathrm{AH}$ and Pugalendi KV: Pharmacological effect of carvacrol on D-galactosamine-induced mitochondrial enzymes and DNA damage by single-cell gel electrophoresis. J Nat Med 65: 568-577, 2011

26. Liu L and Yeh YY: Inhibition of cholesterol biosynthesis by organosulfur compounds derived from garlic. Lipids 35: 2000.

27. Halliwell B and Gutteridge JMC: Oxygen toxicity, oxygen radicals, transition metals and disease. Biochem J 219: 1-14, 1984.
28. Jones $\mathrm{P}$ and Suggett A: The catalase-hydrogen peroxide system. A theoretical appraisal of the mechanism of catalase action. Biochem J 110: 621-629, 1968.

29. Halliwell B: Antioxidant defence mechanisms: from the beginning to the end (of the beginning). Free Radic Res 31: 261-272, 1999.

30. Anandan R, Devi KP, Devaki T and Govindaraju P: Preventive effects of Picrorhiza kurroa on D-galactosamine-induced hepatitis in rats. J Clin Biochem Nutr 25: 87-95, 1998

31. Guha M and Mackman N: LPS induction of gene expression in human monocytes. Cell Signal 13: 85-94, 2001.

32. Kawai T and Akira S: TLR signaling. Semin Immunol 19: 24-32, 2007.

33. Guan KL: The mitogen activated protein kinase signal transduction pathway: from the cell surface to the nucleus. Cell Signal 6: 581-589, 1994.

34. Johnson GL, Dohlman HG and Graves LM: MAPK kinase kinases (MKKKs) as a target class for small-molecule inhibition to modulate signaling networks and gene expression. Curr Opin Chem Biol 9: 325-331, 2005.

35. Kim EK and Choi EJ: Pathological roles of MAPK signaling pathways in human diseases. Biochim Biophys Acta 1802: 396-405, 2010.

36. Klaassen CD and Reisman SA: Nrf2 the rescue: effects of the antioxidative/electrophilic response on the liver. Toxicol Appl Pharmacol 244: 57-65, 2010.

37. Inoue H, Maeda-Yamamoto M, Nesumi A and Murakami A: Delphinidin-3-O-galactoside protects mouse hepatocytes from (-)-epigallocatechin-3-gallate-induced cytotoxicity via upregulation of heme oxygenase-1 and heat shock protein 70 Nutr Res 32: 357-364, 2012.

38. Jaiswal AK: Nrf2 signaling in coordinated activation of antioxidant gene expression. Free Radic Biol Med 36: 1199-1207, 2004.

39. Kensler TW, Wakabayash N and Biswal S: Cell survival responses to environmental stresses via the Keap1-Nrf2-ARE pathway. Annu Rev Pharmacol Toxicol 47: 86-116, 2007.

40. Wu KC, Cui JY and Klaassen CD: Effect of graded Nrf2 activation on phase-I and II drug metabolizing enzymes and transporters in mouse liver. Plos One 7: e39006, 2012. 